\title{
BMJ Open Medical leadership, a systematic narrative review: do hospitals and healthcare organisations perform better when led by doctors?
}

Robyn Clay-Williams, Kristiana Ludlow, Luke Testa, Zhicheng Li, Jeffrey Braithwaite

To cite: Clay-Williams $\mathrm{R}$, Ludlow K, Testa L, et al. Medical leadership, a systematic narrative review: do hospitals and healthcare organisations perform better when led by doctors? BMJ Open 2017;7:e014474. doi:10.1136/ bmjopen-2016-014474

- Prepublication history and additional material for this paper are available online. To view these files, please visit the journal online (http://dx.doi org/10.1136/bmjopen-2016014474).

Received 27 September 2016 Revised 23 August 2017 Accepted 7 September 2017

CrossMark

Australian Institute of Health Innovation, Macquarie University, Sydney, New South Wales, Australia

Correspondence to Dr Robyn Clay-Williams; robyn.clay-williams@mq.edu.au

\section{ABSTRACT}

Introduction Despite common assumptions that doctors are well placed to lead hospitals and healthcare organisations, the peer-reviewed literature contains little evidence on the performance of doctors in leadership roles in comparison with that of non-medical managers.

Objectives To determine whether there is an association between the leader's medical background and management performance in terms of organisational performance or patient outcomes.

Methods We searched for peer-reviewed, English language studies using Medline, Embase and Emerald Management between 2005 and 2017. We included quantitative, qualitative and mixed method empirical studies on the performance of senior healthcare managers where participants were described as doctors or leaders and where comparative performance data were provided on non-medical leaders. Studies without full text available, or no organisational, leadership behaviour or patient measures, were excluded.

Results The search, conducted in Medline $(n=3395)$, Embase $(n=1913)$ and Emerald Management $(n=454)$ databases, yielded 3926 entries. After the application of inclusion and exclusion criteria, 16 studies remained. Twelve studies found that there were positive differences between medical and non-medical leaders, and eight studies correlated those findings with hospital performance or patient outcomes. Six studies examined the composition of boards of directors; otherwise, there were few common areas of investigation. Five interrelated themes emerged from a narrative analysis: the impact of medical leadership on outcomes; doctors on boards; contribution of qualifications and experience; the medical leader as an individual or part of a team and doctors transitioning into the medical leadership role.

Discussion and conclusion A modest body of evidence supports the importance of including doctors on organisational governing boards. Despite many published articles on the topic of whether hospitals and healthcare organisations perform better when led by doctors, there were few empirical studies that directly compared the performance of medical and non-medical managers. This is an under-researched area that requires further funding and focus.

\section{Strengths and limitations of this study}

- To the best of our knowledge, this is the first systematic review of the literature published over the last decade to determine whether healthcare leaders who are doctors perform better than those with non-medical backgrounds in terms of organisational performance or patient outcomes.

- We developed robust search strategies and a rigorous reviewing process aiming to minimise bias and ensure the objectiveness and transparency of the systematic review.

- A modest body of evidence supports the importance of including doctors in the composition of governing boards to improve organisational performance.

- There were insufficient studies meeting inclusion criteria to enable our research question to be fully answered.

\section{INTRODUCTION \\ Rationale}

Prior to the 1970s, doctors very often ran hospitals $^{12}$ and administrators played a subordinate, coordination, rather than a leadership, role. As healthcare moved towards a more business-bureaucratic ${ }^{3}$ model of practice, administrators were engaged to manage general organisational-operational business performance, but doctors continued to expend substantial resources and manage the major decisions affecting patient care. In 1983, the Griffiths Report ${ }^{4}$ was released in the UK, paving the way for the introduction of a new purchaser-provider model of healthcare. In the USA, the idea which became known as clinical directorates was established. ${ }^{5}$ The Medical Director (MD) role and clinical directorates became more widely established and, over time, enabled more senior managers to have greater control over resources. Doctors did not always consider such roles attractive ${ }^{5}$ but they felt that they needed to take up these part-time appointments in order to continue to partake in decisions affecting their work. 
In the 1990s, when managed care was established in the $\mathrm{USA}^{6}{ }^{6}$ new drivers emerged as doctors moved from parttime advisory roles into full-time management in order to contribute to running healthcare organisations, secure greater control over resource allocation and participate in senior decision-making.

Today, doctors are well established in management roles, with the first survey of Accountable Care Organisations (ACOs) in the USA finding that by $2014,51 \%$ were led by doctors. ${ }^{7}$ Within hospitals and other healthcare organisations, medical departments are normally led by doctors, and they report to the MD (or equivalent) who is typically a member of the executive team (see Dwyer ${ }^{8}$ for a literature-based review on the roles of medical managers). By the turn of the 21st century in the USA, $50 \%$ of physician executives no longer practised medicine, ${ }^{6}$ and physician executives began to gain acceptance as administrators of managed care institutions. ${ }^{910}$ In the $\mathrm{UK}^{211}$ (and elsewhere ${ }^{112}$ ), where the culture was historically less well disposed to accepting doctors who relinquish their clinical work, ${ }^{13}$ the majority of medical executives today act as 'hybrid managers', who continue to manage a clinical workload alongside their management responsibilities. In the UK National Health Service (NHS), where doctors hold positions of power within healthcare organisations that enable them to participate in managerial decisions, enhancing medical engagement in leadership is seen as a factor that may contribute to improved organisational performance. ${ }^{11}$ Benefits to employing doctors in healthcare management roles may include bottom-up leadership, greater political influence and improved communications between doctors and senior management. ${ }^{14}$ Current focus on engaging doctors in leadership centres on efforts to link clinical decisions with those of strategic management, and it has broadened to include key accountabilities for quality of care in addition to resource management. ${ }^{15}$

Today's leaders in healthcare perform many tasks. For this review, we have adopted the UK's King's Fund description of the healthcare leadership task: 'to ensure direction, alignment and commitment within teams and organisations'. ${ }^{16}$ This task may incorporate elements of leadership, management and administration.

Despite the prevalence of such physician executives (to use the US term) occupying leadership roles in health systems, we do not know to what extent prior medical training or experience as a doctor affects the performance of healthcare executives. Research questions include: do healthcare executives who are doctors perform better than those who are not, and, if so, in what ways do they perform better? What is it about a doctor that might enhance their leadership ability: medical training, experience in the medical role or something else? Is it important for organisational performance to have doctors as members of the executive leadership team, and if so, why?

Previous research has found no difference in performance between medical and non-medical managers ${ }^{17-19}$; however, opinion on the topic of "which profession should manage hospitals' is prevalent and polarised. There appears to be a view that the doctor's mindset is different from that of the general healthcare manager. ${ }^{20}{ }^{21}$ Arguing against the benefits of medical leadership, papers cite doctors' overidentification with their professional clinical role, their tendency to be conservative individualists rather than team players, their lack of formal management training and their purported weaknesses in financial management and organisational strategy. ${ }^{122-24}$

However, doctors prefer to be led by doctors, ${ }^{11}$ and articles in favour of medical leadership cite doctors' strengths in addressing patient outcomes, quality and safety issues and decision-making and point to their ability to specialise and intelligence. A recent study surveying doctors' reactions to hospital reform found that doctors who were also leaders reacted more positively to hospital reform than those who spent most of their time caring for patients. ${ }^{25}$ Clearly, the question 'Do hospitals and healthcare organisations perform better when led by doctors?', particularly in relation to the leadership structures of modern healthcare systems, has not been settled.

\section{Objectives}

This study presents the results of a systematic review of the literature published since 2005 on medical leadership. We sought contemporary evidence on the leadership performance of executives or senior managers who were also doctors. The objective of the review was to determine if there is an association between leaders having a medical background and management performance, in terms of organisational performance or patient outcomes. This objective was framed by the research question: do hospitals and healthcare organisations perform better when led by doctors?

To enable us to objectively answer our question, and to minimise the confounders associated with comparisons in healthcare, we sought quantitative, qualitative and mixed method empirical studies reporting on leadership performance that included medical and non-medical leaders in the same setting.

\section{METHODS}

\section{Eligibility criteria}

Types of participants

We included empirical studies on senior managers in healthcare organisations that involved participants who were both doctors and leaders and participants who were non-medical leaders. Non-medical leaders included those who had a clinical background other than medicine (eg, nurses, allied health professionals) and those who did not have a clinical background.

\section{Types of outcomes}

After examining the literature, we included three types of outcome measures. These were (1) patient measures (eg, patient outcomes or processes of care), (2) organisational 
Table 1 Medline, Embase and Emerald Management search strategies

\begin{tabular}{|c|c|c|}
\hline Database & Medline & \\
\hline Strategy & Searches & Results \\
\hline$\# 1$ & $\begin{array}{l}((\text { executive } \$ \text { or leader } \$ \text { or leadership\$ or manager } \$ \text { or director } \$ \text { or } C E O \text { or board } \$ \text { ) adj3 (physician\$ } \\
\text { or doctor } \$ \text { or clinician\$)).ab. }\end{array}$ & 4158 \\
\hline \#2 & Limit \#1 to (English language and yr="2005 - Current”) & 2037 \\
\hline \#3 & *Physician Executives/ & 4069 \\
\hline Database & Embase & \\
\hline Strategy & Searches & Results \\
\hline$\# 1$ & $\begin{array}{l}\text { ((executive } \$ \text { or leader\$ or leadership\$ or manager\$ or director\$ or CEO } \$ \text { or board\$) adj3 (physician\$ } \\
\text { or doctor\$ or clinician\$)).ab. }\end{array}$ & 5766 \\
\hline Strategy & $\begin{array}{l}\left(\left(\text { executive }^{\star} \text { or leader }^{*} \text { or leadership* or manager }{ }^{\star} \text { or director }{ }^{\star} \text { or CEO* or board*) and (physician* or }\right.\right. \\
\left.\left.\text { doctor }^{\star} \text { or clinician }{ }^{\star}\right)\right) \text {.ab. Limit publication date to January } 2005-\text { June } 2017\end{array}$ & 454 \\
\hline
\end{tabular}

measures (eg, staffing, finance or hospital ratings) and (3) leadership behaviour measures (eg, management processes, teamwork or decision-making).

\section{Information sources}

We searched for peer-reviewed, English language studies using three academic databases: Medline, Embase and Emerald Management. The search was limited to empirical research published between 1 January 2005 and 7 June 2017.

\section{Search}

The search was designed, in collaboration with a professional research librarian, to capture both the executive leadership and the medical practitioner role. Seven terms were included for executive leadership and linked using the Boolean operator OR to maximise the sensitivity of the search: 'executive', 'leader', 'leadership', 'manager', 'director', 'CEO' or 'board'. For the role of medical practitioner, terms including 'physician', 'clinician' and 'doctor' are used sometimes interchangeably in the literature. Thus, we searched for all three terms using the OR operator. The searches were combined and refined using a proximity operator. Full-search strategies are presented in table 1.

\section{Study selection}

Search results were aggregated and imported to an EndNote library, and duplicate entries were removed. Pairs of reviewers (RC-W:LT; KL:ZL) cross-checked 110 (approximately 3\%) of the citation titles and abstracts in a double review in order to establish inter-rater reliability. Articles were excluded based on the following criteria: pre-2005; language other than English; non-peer-reviewed literature; setting other than healthcare; non-primary research including systematic reviews and does not include participants who are both doctors and leaders. Any discrepancies between the reviewers were discussed until a consensus was reached. The remaining citations were randomly assigned to the four reviewers who independently assessed titles and abstracts against the exclusion criteria, with regular discussions held at team meetings. While we did not include literature reviews, we snowballed the reference section of any review identified, searching for additional papers that might meet inclusion criteria. The selected articles were then subject to a full-text review where further criteria were added to the exclusion criteria: full-text unavailable; neither medical background/training nor leadership is assessed as a variable in the data analysis; does not include organisational and/or patient outcomes.

\section{Data collection process and data items}

Data from the included studies were extracted into a locally developed form for analysis. Elements extracted were (A) the full reference; (B) location; (C) language; (D) period of data collection; (E) study type; (F) study primary and secondary aims; $(\mathrm{G})$ exclusion criteria; $(\mathrm{H})$ data (total number of organisations, type of organisations, data types and sources used to performance and/or outcomes, methodological/statistical approach to identify performance and/or outcomes); (I) methods (methods used to study contextual/success factors associated with medical leaders, eg, interview, survey, observation), participants and data analysis methods; (J) findings (quantitative results and qualitative results or contextual factors most important for explaining relationship between medical background of leader and performance) and (K) implications.

\section{Risk of bias}

Risk of bias within studies was assessed using criteria developed by Hawker et al. ${ }^{26}$ Ratings were assigned (poor, fair, 
good) across nine different categories: (1) abstract and title, (2) introduction and aims, (3) method and data, (4) sampling, (5) data analysis, (6) ethics and bias, (7) results, (8) transferability or generalisability and (9) implications and usefulness. Risk of bias potentially affecting the cumulative evidence across studies was determined by examining study methods, ethics committee approvals, study funding and authors' conflicts of interest.

\section{Synthesis of results}

Results were analysed through a narrative synthesis of extracted data. ${ }^{27}$ Extracted data were coded and organised to explore connections between data elements and to develop sets of concepts. Segments of data were then linked in a formal fashion to determine relationships that may exist between different data elements and allow themes to emerge.

\section{RESULTS}

\section{Study selection}

The search results and review process are presented in figure 1, using the Preferred Reporting Items for Systematic Review and Meta-analyses (PRISMA) flow chart. The search performed on Medline $(\mathrm{n}=3395)$, Embase $(\mathrm{n}=1913)$ and Emerald Management $(\mathrm{n}=454)$ yielded a total of 3926 articles after the removal of 1849 duplicates. To test inter-rater reliability, we used Cohen's kappa and found high levels of agreement between the paired reviewers for the $3 \%$ double review, $\mathrm{K}=0.78(\mathrm{p}<0.0001)$ for pair 1 and $\mathrm{K}=0.88 \quad(\mathrm{p}<0.0001)$ for pair 2 . The remaining screening of title and abstract resulted in 113 studies eligible for full-text review. The full-text review stage leads to the inclusion of 22 quantitative, qualitative or mixed method studies in the penultimate pool. After an additional exclusion criterion was added, requiring that included studies provide data to allow a comparison of medical and non-medical leadership, eight more studies were eliminated. The literature search identified five literature reviews on topics associated with medical leadership. ${ }^{81428-30}$ The references of these five reviews were searched for additional studies that met inclusion criteria. Two additional studies were identified as a result of this process, resulting in a final inclusion of 16 studies.

\section{Study characteristics}

The characteristics of the 16 studies that met inclusion criteria are presented in online supplementary table 1 . Fourteen studies conducted quantitative analysis: ten studies analysed questionnaire survey data ${ }^{71-39}$; one study (Jiang $e a^{33}$ ) also provided processes of care data, and another provided hospital performance data (Saleh et $\left.a l^{88}\right)$. Two studies analysed US Statewide Health Planning and Development data from California, ${ }^{40}{ }^{41}$ one study analysed data from the AMADEUS database and hospital and insurance documents ${ }^{42}$ and another study analysed UK hospital trust data. ${ }^{43}$ One study conducted a qualitative analysis of interview and observation data, ${ }^{44}$ and another conducted a mixed method analysis, ${ }^{45}$ which combined findings from a review of hospital documents with qualitative analysis of interviews with hospital Chief Executive Officers (CEOs) and board members. Seven studies were from the USA; the remainder were from Finland (2), Germany (2), the UK (1) Ireland (1), Norway (1), Lebanon (1) and Australia (1).

\section{Results of individual studies}

Six studies reported on the performance of hospital boards. ${ }^{33} 40-4345$ Veronesi $e t a l^{43}$ examined the impact of clinicians appointed to the boards of 102 English NHS hospital trusts on quality of hospital care provided from 2006 to 2009. Composition of boards was determined from hospital trust annual reports. Compliance with health and well-being, clinical effectiveness, safety and patient focus, and ease and equity of access care standards was obtained from the UK Healthcare Commission and Dr Foster (a commercial provider of healthcare benchmarking data). A greater percentage of doctors on boards was associated with a better-quality rating of service providers. Trusts achieving a four rating had an average of $15.01 \%$ of directors with a medical background, whereas in trusts achieving only a one rating, $11.09 \%$ board directors were doctors. This finding was confirmed in relation to lower morbidity rates and tests to exclude the possibility of reverse causality, whereby doctors joined the boards of better performing trusts. No equivalent association was found for clinical professions such as nurses and other allied health professions.

De Andrade $^{41}$ investigated whether having board members with medical expertise in 281 USA hospitals affected the levels of uncompensated care provided. A quantitative analysis of data from the California Office of Statewide Health Planning and Development between 1997 and 2010 found that doctors' board membership was not related to provision of uncompensated care, except when the hospital's ownership status was taken into account. Relative to non-profit and public hospitals, for-profit hospitals provided more uncompensated care, the higher the percentage of doctors on the board. For an average for-profit board size of 10 members, substituting one member by a doctor increased the amount of uncompensated care provided by $19 \%$.

On the theme of boards, Prybil ${ }^{45}$ sought to determine whether high-performing and mid-range hospitals differ in board structures, processes and practices. High-performance hospitals included at least three of the Solucient Center for Healthcare Improvement's '100 Top Hospitals' in 1999-2003. A mixed method analysis of hospital documents and interviews with hospital CEOs and board members was conducted for seven matched pairs of USA hospitals, finding that doctors form a larger component of the boards of high-performing hospitals $(30.3 \%)$ than of mid-range hospitals $(20.8 \%)$. Doctors comprised $25 \%$ or more of the boards' voting members in five of the seven high-performing hospitals but only one mid-range hospital. 


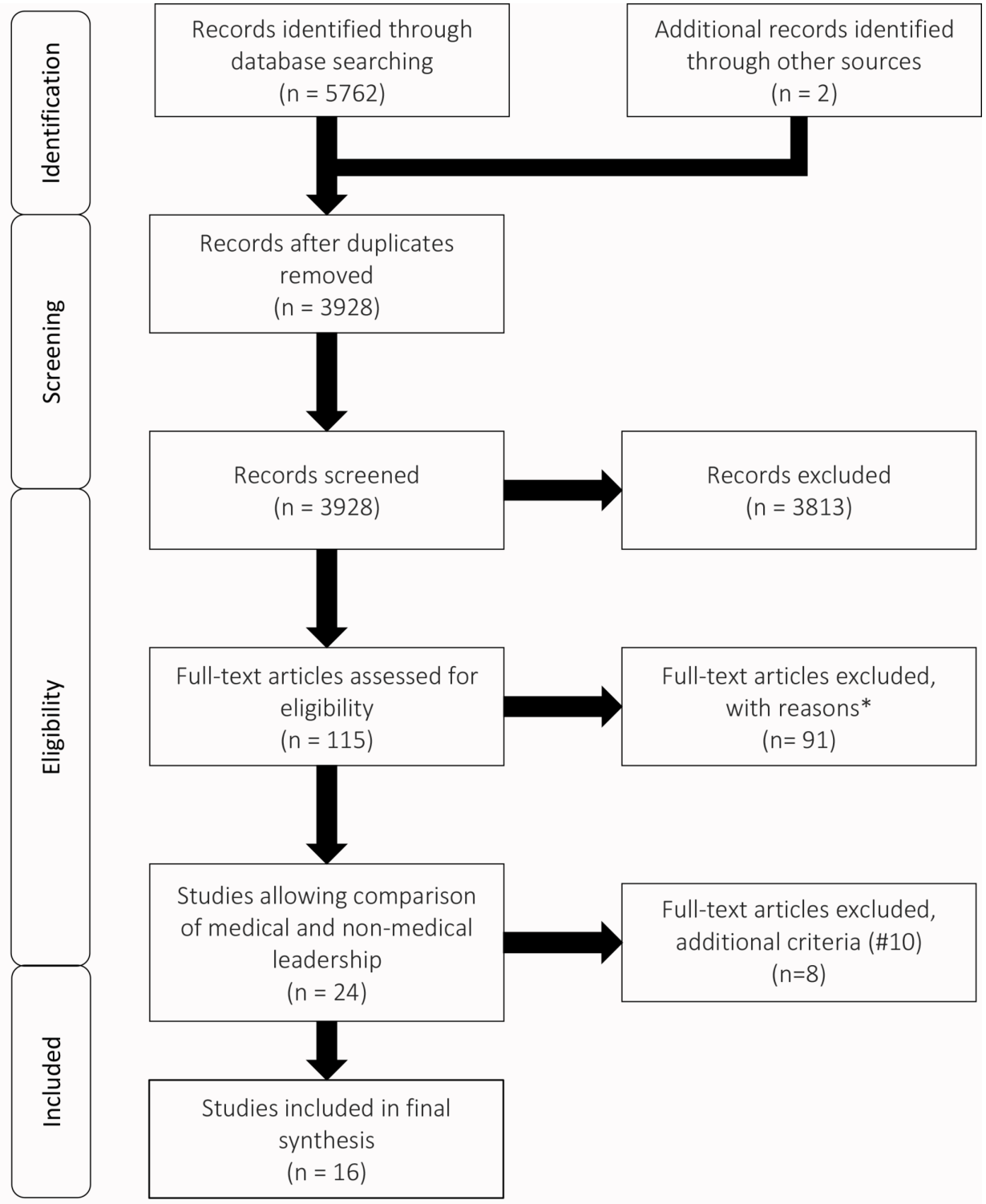

\begin{tabular}{lll} 
*Legend \\
\multicolumn{2}{l}{ Reason for exclusion } & $\begin{array}{l}\text { Number of } \\
\text { studies* }\end{array}$ \\
\hline 1 & Pre-2005 & 0 \\
2 & Other languages & 0 \\
3 & Not peer-reviewed literature & 0 \\
4 & Setting other than healthcare & 0 \\
5 & Full-text not available & 8 \\
6 & Not primary research (including systematic reviews) & 4 \\
7 & $\begin{array}{l}\text { Does not include participants who are both doctors or } \\
\text { leaders }\end{array}$ & 20 \\
8 & $\begin{array}{l}\text { Neither medical background/training or leadership is } \\
\text { assessed as a variable in the data analysis }\end{array}$ & 18 \\
9 & $\begin{array}{l}\text { Does not include organisational and/or patient } \\
\text { outcomes }\end{array}$ & 64 \\
10 & $\begin{array}{l}\text { Does not include medical and non-medical leaders in } \\
\text { the study }\end{array}$ & 8 \\
\hline
\end{tabular}

*The numbers do not add up to the total number of studies excluded because a proportion of studies were excluded based on multiple criteria.

Figure 1 Search and review strategy (PRISMA flow diagram). 
Jiang et $a l^{33}$ examined whether differences exist in quality performance of 490 USA hospitals in relation to adoption of particular practices in board oversight of quality. Data consisted of (1) a survey of 562 hospital CEOs on board practices, (2) process of care measures for three clinical conditions (heart attack, heart failure, pneumonia) and (3) outcomes measures, consisting of risk-adjusted mortality rates, for the same three conditions. Sixty per cent of participating hospitals had a Chief Medical Officer or Vice President of Medical Affairs on the committee; this resulted in significantly higher process of care scores $(85.3 \%$ vs $81.0 \%$, $\mathrm{p}<0.05)$ and lower risk-adjusted mortality rates $(5.6 \%$ vs $7.3 \%, \mathrm{p}<0.05)$ than hospitals that did not have a Chief Medical Officer or Vice President of Medical Affairs as a committee member. Eighty-three per cent of participating hospitals had medical staff on the committee; this resulted in significantly higher process of care scores $(84.2 \%$ vs $80.9 \%, \mathrm{p}<0.05)$ but no difference in risk-adjusted mortality rates. Sixty-three per cent of participating hospitals had a clinical board member on the committee; this resulted in no difference in process of care scores but significantly lower risk-adjusted mortality rates $(5.7 \%$ vs $7.2 \%, \mathrm{p}<0.05)$.

Bai and Krishnan ${ }^{40}$ examined whether hospitals without medical participation on their boards of directors delivered lower quality of care in 142 non-profit hospitals in the USA. Quantitative data were obtained from the US Hospital Quality Alliance and the California Office of Statewide Health Planning and Development. The study found that boards without medical members were associated with a decrease of three to five percentage points in quality of care for heart failure, pneumonia and surgery infection prevention.

Moving to Germany, Kuntz et $a l^{42}$ examined differences in the financial performance of hospitals with regard to ownership by studying the size and composition of supervisory boards in 175 hospital companies operating 246 hospitals (14\% of all German acute care hospitals in 2009). The study reported on a quantitative analysis of hospital financial performance data (from the AMADEUS database) and information on hospital and board characteristics (from business and quality reports, hospital websites and health insurers). Data were obtained from all participants in 2009 and from a subsample of 163 hospital companies in 2010. Financial performance was based on four measures: return on assets (ROA), earnings before interest and tax margin, total profit margin and net income. Doctors comprised, on average, $11.7 \%$ board members. Financial performance and board size and composition depended on ownership ( $<<0.01$ for ROA and $p<0.001$ for the other four performance measures). An increase in board size and greater political participation were negatively associated with all five tested measures of financial performance, an increase in nurse and economist participation was negatively associated with financial performance and no associations were found for clerical participation. An increase in physician participation, however, was positively associated with a $5 \%$ increase in ROA ( $\mathrm{p}=0.061)$.

Two studies reported on doctors' involvement in strategic decision-making. ${ }^{3738}$ Parayitam et a l $^{37}$ examined the self-reported outcomes of decisions when physician executives were involved in strategic decision-making processes in 109 USA hospitals. Hypotheses were that increased numbers of doctors involved in strategic decision-making teams would be associated with better decisions, greater understanding of the rationale of decisions and more commitment to decisions. The sample of 114 CEOs and 254 strategic decision makers (executive officers, director of human resources, chief technical offices, chiefs of staff, personnel involved in facilities, maintenance) completed a survey reporting their decision quality, understanding and commitment. Structural equation modelling of the data suggested that the ratio of doctors was positively correlated with decision understanding, commitment and quality.

Saleh $e t a l^{38}$ explored the use of strategic planning processes in $79(56.4 \%)$ Lebanese hospitals and investigated its association with financial performance. Hypotheses included that the level of physician involvement in the strategic planning process is positively associated with hospital performance. Quantitative analysis of survey data on hospital-reported participation in strategic planning processes and hospital performance data from the Lebanese Ministry of Public Health (occupancy rate and revenue per bed) found that there was no association between the level of physician involvement in the strategic planning process and hospital outcomes; generally, physician involvement was low (4.1 out of a possible score of 7$)$.

Two studies reported on medical leadership of organisations. ${ }^{732}$ In the USA, Colla $e t a l^{7}$ measured how doctor-led organisations compared with other ACOs in terms of structure, size and care provided and explored the degree of doctor engagement in managing ACOs. ACOs are groups of providers that are jointly responsible for caring for a nominated population of patients. Fifty-one per cent of the 173 ACOs in the study self-identified as doctor-led, $33 \%$ self-identified as jointly led by hospital and doctor and the remainder were led by hospitals or other entities. Doctor-led ACOs were found to be more likely to have advanced IT capabilities and better outpatient care than non-doctor-led ACOs and were more likely to measure and report financial performance at practice and clinicians levels; however, this finding was confounded by the fact that doctor-led ACOs are less likely to include hospitals and more likely to include physician groups. Performance of ACOs was not assessed; therefore, it was not possible to determine whether performance was related to whether leaders were doctors or non-medical managers.

Goodall $^{32}$ examined the relationship between hospital performance and whether the CEO was a doctor or a non-medical manager. Hospital performance was determined by media-generated league tables, produced by 
US News and World Report's 'Best Hospitals' in 2009. Three hundred healthcare executives from three specialties (cancer, digestive disorders, heart and heart surgery) were surveyed in the top 100 USA hospitals. Positive association was found between physician CEOs and hospital performance for all three hospital specialties $(p<0.001)$. While higher-performing hospitals were associated with physician CEOs, causation was not able to be determined (eg, higher-performing hospitals may just prefer to have doctors as leaders).

Five studies reported on medical leadership within organisations. ${ }^{31} 34353944$ Konu and Viitanen ${ }^{34}$ investigated the incidence of shared leadership among 433 middlelevel managers (eg, chief doctors, nursing directors) in social service and healthcare in Finland. Quantitative analysis of survey data on leadership practices found that shared leadership practices were more common among managers without a medical background.

Another study sought to determine whether evaluations on the impact of knowledge sources affecting their decision-making differ depending on the manager's professional background, activity sector, gender, management experience or age, among 404 middle-level social and healthcare managers in a Finnish hospital (Simonen et $a l^{39}$ ). Quantitative analysis of survey data revealed that managers who were also doctors more strongly perceived that their decision-making was influenced by their own professional experience, journals and scientific research within their own professional field and nationwide interaction within their own profession. Differences were found between doctor managers and nurse managers with respect to organisation documents and publications, which carried more weight in nurse managers' decision-making. No differences were found between managers of different professional backgrounds regarding other knowledge sources.

In other work, Agarwal et $a l^{31}$ sought to determine whether there is a positive association between Management Practices Score (MPS) and the level of clinical education of managers in 42 Australian acute care public hospitals. The MPS for each hospital was calculated from interview responses of 116 managers to a survey of 21 hospital management practices across multiple dimensions. The study found no association between the number of doctors in each hospital and the MPS $(\mathrm{p}=0.779)$. The coefficient on the level of skills and education within hospitals, however, was positive and significant $(\mathrm{p}=0.06)$, indicating that hospitals with a higher proportion of clinically qualified and skilled managers perform significantly better in management practices.

Spehar et $a l^{4}$ investigated how clinicians' professional background influences their transition into the managerial role and identity as clinical managers in four public hospitals and two health trusts in Norway. Interviews were conducted with 30 clinician managers (13 doctors), 20 of these were also observed in management and staff meetings during the day. Qualitative analysis of interview and observation data revealed that doctors experienced difficulties in reconciling the clinical and management role and used clinical work to gain legitimacy and respect from medical colleagues. In contrast, nurses experienced a faster and more positive transition into the manager role and were more fully engaged in the managerial aspects of the role.

Moving countries again, Kuntz et $a l^{35}$ assessed the relationship between the amount of medical involvement in leadership and staff-to-patient ratios. The study was conducted in Germany, where hospitals are managed by an executive leadership team consisting of a commercial director, an MD and a nursing director. The study was controlled for the size and case mix of the 604 participating hospitals, whether they were public or private, the degree to which they were rural and whether the doctors were salaried or contracted. High staff-to-patient ratios for both nurses and doctors are associated with better hospital performance. ${ }^{4647}$ The study found a relationship between full-time MD or heavily involved part-time MD and a higher staffto-patient ratio. Full-time MDs significantly improved the staff-to-patient ratios for both doctors and nurses (doctors: 1.96, $\mathrm{p}<0.01$; nurses: 4.44, $\mathrm{p}<0.01$ ), whereas part-time MDs only improved the staff-to-patient ratios for doctors (eg, an increase of part-time involvement from $15 \%$ to $25 \%$ resulted in an increase of 2.49 doctors per thousand inpatients).

Staying in Europe, O'Keefe ${ }^{36}$ measured and compared risk aversion in 788 Irish clinicians, clinical managers, non-clinical managers and non-clinical public representatives, in terms of willingness to discharge a patient from the emergency department. The study found no significant difference between clinicians and clinician managers but found significant differences between clinicians and non-clinicians (including between clinical and non-clinical managers), with the non-clinical participants being more risk averse. Limitations included the following: (1) there was a large variation in risk tolerance, even between clinicians; (2) it was a single study in a single country; and (3) clinicians are likely to be more familiar both with actual events depicted in the scenarios and also with the process of making treatment choices that may result in death. The study did not provide indication of an objectively appropriate level of risk, but the authors suggested that the clinicians had a more pragmatic approach to decision-making.

\section{Risk of bias}

While studies were generally well designed and executed, generalisability and implications, and usefulness of the findings, was low (table 2). ${ }^{26}$ There was a risk of bias across studies, in that the majority (12) studies collected self-reported data on aspects of medical management, for example, surveys or interviews, rather than objective data. Many of the studies did not report ethics approval, study funding or authors' conflicts of interests. 


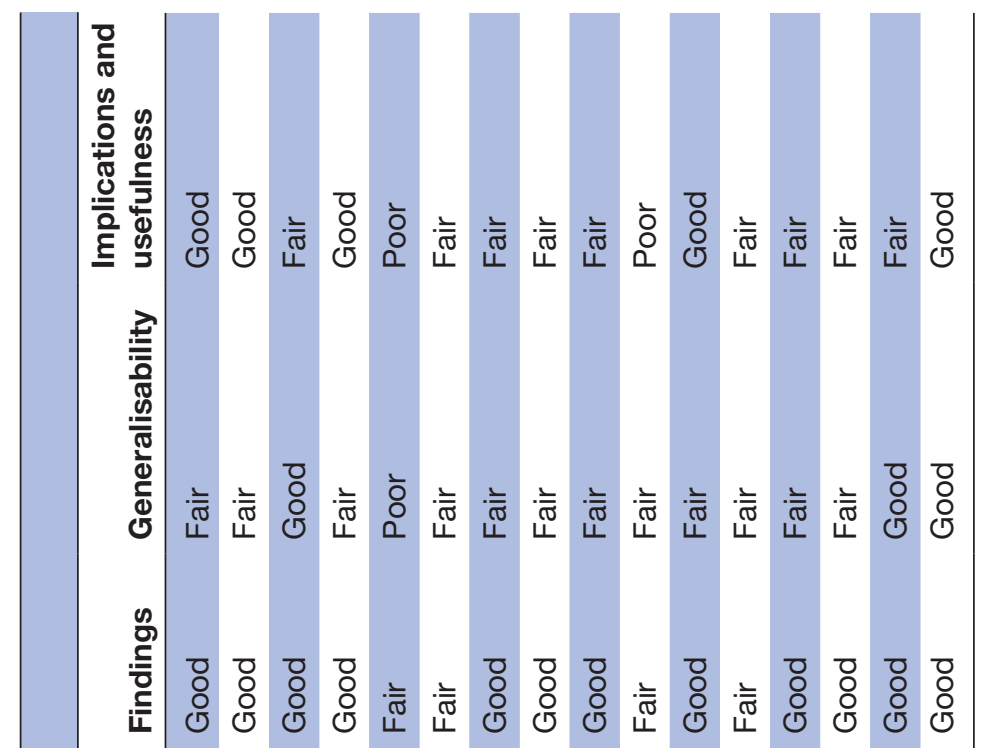

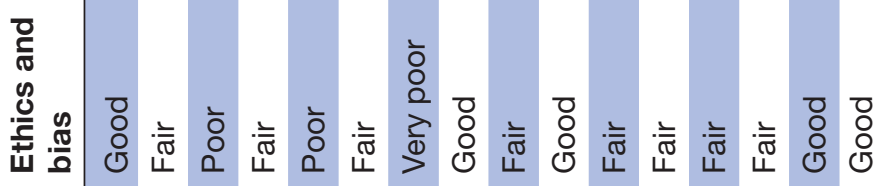

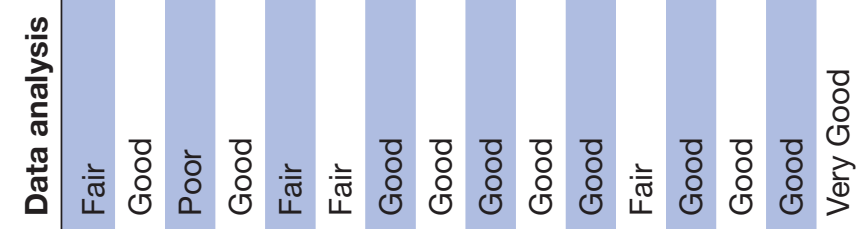

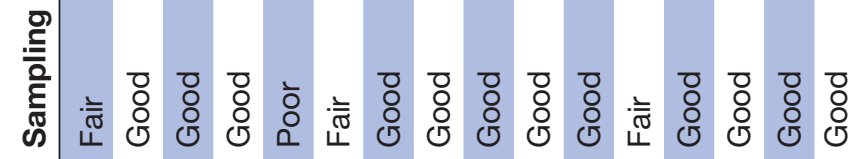

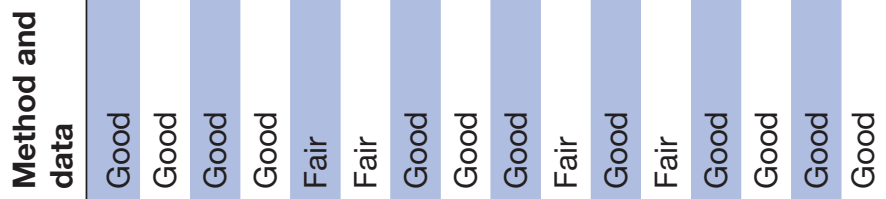

垔

कृ

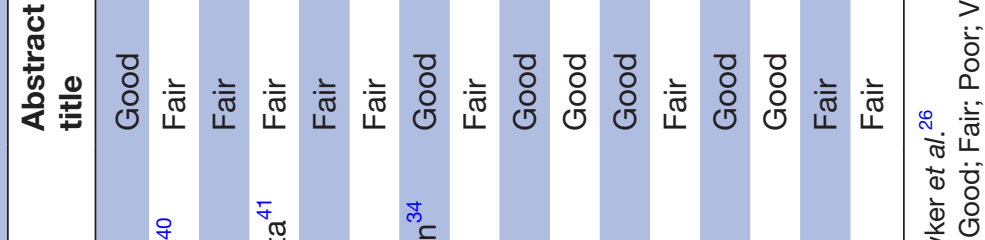

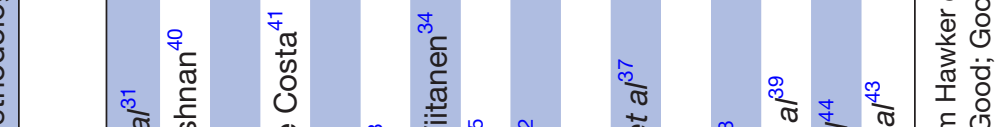

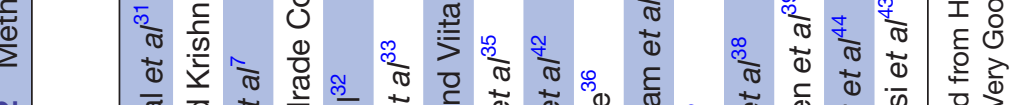

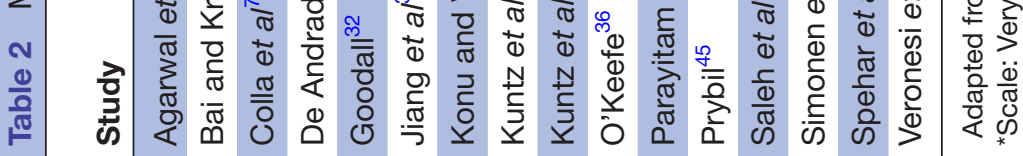




\section{Study synthesis}

Extracted data were cross-checked for accuracy and coded by four researchers (ZL, KL LT and RC-W). One researcher (RC-W) completed a narrative synthesis of the coded data, by linking the data to form five inter-related themes: impact of medical leadership on outcomes; doctors on boards; contribution of qualifications and experience; the leader as an individual or part of a team; and doctors transitioning into the medical leadership role. Four broad categories of articles were found in the literature search: (1) individual perspectives on medical leadership (the majority of excluded articles), frequently based on personal experience; (2) empirical research based on self-reported data about medical leadership (surveys, interviews, focus groups); (3) objective empirical research on the role or characteristics of healthcare leaders (observations, other data) and (4) objective empirical research on the relationship between medical leaders and outcomes (hospital performance data, patient outcomes).

\section{DISCUSSION}

\section{Summary of evidence}

Themes

Impact of medical leadership on outcomes

Given the complexity encountered within modern healthcare organisations, ${ }^{48}$ it is difficult to demonstrate the impact of leadership on outcomes-even when assessing medical and non-medical leadership in the same setting. Four of the 16 studies showed either no difference or a negative relationship between medical and non-medical leadership in the aspects of performance that they investigated. ${ }^{34} 383944$ The remaining 12 studies found that there were differences between medical and non-medical managers, and eight of these studies ${ }^{732} 3340-4345$ correlated findings with hospital performance or patient outcomes. The studies did not provide sufficient information that would allow us to determine why medical leadership might make a difference. Other than board composition (discussed below), there were few common areas of investigation, with studies examining varied topics: risk aversion, IT adoption, patient care arrangements, financial reporting, staff-to-patient ratios and so on.

\section{Doctors on boards}

We found that evidence supporting the relationship between doctors' board participation and organisational performance is accumulating, with empirical studies from the UK, ${ }^{43}$ Europe $^{42}$ and the USA ${ }^{33} 404145$ all reporting positive associations. This finding is consistent with pre-2005 data $^{49} 50$ and supported by a recent literature review on the topic. $^{30}$

\section{Contribution of qualifications and experience}

None of the studies provided data on the characteristics of medical leaders that were associated with higher performance. We know that doctors who receive leadership or management training may perform well in leadership roles. Xirasagar et $a l,{ }^{51}{ }^{52}$ for example, when examining the relationship between leadership styles, training and effectiveness of doctors who are managers, concluded that doctors who completed graduate managerial training such as Masters of Business Administration, Masters of Health Administration or Masters of Public Health degrees, or more than 30 days of in-service training, were likely to be more effective leaders. In the included studies, there was no demographic information on the leaders who participated in the study to enable us to disentangle the contribution to the findings of medical qualifications, medical experience or management experience. While our included studies found both positive ${ }^{43}$ and negative ${ }^{34} 3944$ differences between a leader's medical and nursing background in relation to leadership outcomes, much of the literature conflates doctors and nurses into a single group of 'clinicians'. ${ }^{29}$

\section{Medical leader as an individual or part of a team}

Only two studies ${ }^{72}$ explored the performance of the doctor as the CEO or primary organisation leader, and both found that there was an association between medical leadership and organisational performance. The remaining studies explored outcomes when a doctor was included as part of the leadership team. While not providing direct evidence supporting the doctor as the leader, they emphasise current thinking about the need to engage doctors in healthcare leadership to improve organisational culture and patient outcomes. ${ }^{53}{ }^{54}$ In a comparison of six high-performing and low-performing UK trusts, for example, Mannion et al found that poor-performing organisations were characterised by cultures where a few senior consultants exerted a disproportional influence over organisational priorities. ${ }^{55}$ Doctors who are engaged as part of a leadership team are often hybrid managers, who retain a clinical role, and studies elsewhere have found these managers less likely to be effective in their non-clinical leadership role. ${ }^{56}$ Kippist and Fitzgerald, ${ }^{12}$ for example, found that hybrid managers would prioritise clinical work over management, leading to additional burden on their managerial colleagues, thereby questioning the effectiveness of the hybrid clinician manager. Spehar et $a l^{24}$ explored influences and strategies employed by 30 hybrid leaders in four hospitals in Norway and concluded that doctors who were managers could not influence other doctors without drawing on professional power and that doctors felt they had to maintain their clinical skills to retain credibility among peers. This emphasis on professional skill constrained doctors from drawing effectively on positional power. This expert power was not retained and had to be continuously regenerated. In addition, rather than collaborating, doctors saw clinician managers of other departments as competitors and saw themselves as representatives of their own professional group. 


\section{Doctors transitioning into the medical leadership role}

The five studies reporting on medical leadership within organisations touched on some of the barriers or enablers ${ }^{57}$ for doctors seeking to enter management, including difficulties reconciling the clinical and management role. Professional and organisational culture strongly influences the roles that practitioner leaders can take up and the influence they can wield. ${ }^{58}$ Ham $e t a \rho^{59}$ investigated, via 22 qualitative interviews, the experiences of doctors who become Chief Executives of UK NHS organisations and found that medical managers tended to be 'keen amateurs' rather than trained managerial professionals. Kisa and Ersoy, ${ }^{60}$ via a 31 -item time management questionnaire, found that medical managers have poor time management skills. Medical managers are not usually trained in leadership, ${ }^{1159}$ which may explain both these findings and some of the negative perceptions of doctors as managers. There are also some indications within our included studies that doctors should adopt a more multidisciplinary approach to be effective leaders. ${ }^{34} 3944$ West and Barron ${ }^{61}$ found that medical managers consult or network mostly with other medical managers and that it is the non-medical managers who act as brokers between professional groups. This finding is supported by other work in the field. ${ }^{62}$

\section{Limitations}

Our review was limited in date range and language (English only), and we found insufficient studies meeting inclusion criteria to enable our research question to be robustly answered, hence the decision to craft a narrative review. Most studies examined only one or two aspects of leadership because these aspects were different across studies, it was not possible to generalise the findings. Due to the diversity of keywords and publication venues used by authors of studies on medical leadership, it was difficult to ensure that all relevant literature has been captured by our search strategy. It is also important to consider that risk of bias was evident across studies due to the majority of studies employing self-reported measures and an absence of information concerning ethics approval, funding or conflicts of interests in some studies.

\section{CONCLUSIONS}

There is a modest body of evidence supporting the importance of including doctors in the composition of hospital or organisational governing boards. Despite a large volume of published literature on the topic of whether hospitals and healthcare organisations perform better when led by doctors, however, there are few studies that have examined this topic in a robust way or directly compared the performance of medical and non-medical managers. While we found 16 studies that provided empirical data in respect of this question, only two studies explored the role of organisational leader or CEO. This is an under researched area that requires further funding and focus.
Contributors RC-W and JB conceptualised the study. ZL, KL and LT conducted the database searches. RC-W, KL, ZL and LT conducted the search and review strategy. $\mathrm{RC}-\mathrm{W}$ drafted the manuscript. All authors reviewed the manuscript for critical content.

Competing interests None declared.

Provenance and peer review Not commissioned; externally peer reviewed.

Data sharing statement The full dataset is available from the corresponding author on request.

Open Access This is an Open Access article distributed in accordance with the Creative Commons Attribution Non Commercial (CC BY-NC 4.0) license, which permits others to distribute, remix, adapt, build upon this work non-commercially, and license their derivative works on different terms, provided the original work is properly cited and the use is non-commercial. See: http://creativecommons.org/ licenses/by-nc/4.0/

(c) Article author(s) (or their employer(s) unless otherwise stated in the text of the article) 2017. All rights reserved. No commercial use is permitted unless otherwise expressly granted.

\section{REFERENCES}

1. Bruce A, Hill S. Relationships between doctors and managers: the Scottish experience. J Manag Med 1994;8:49-57.

2. Buchanan D, Jordan S, Preston D, et al. Doctor in the process. The engagement of clinical directors in hospital management. J Manag Med 1997;11:132-56.

3. Davies HT, Harrison S. Trends in doctor-manager relationships. BMJ 2003;326:646-9.

4. Griffiths R. NHS management inquiry: report to the secretary of state for social services. Department of Health and Social Security, London 1983.

5. Heyssel RM, Gaintner JR, Kues IW, et al. Decentralized management in a teaching hospital. N Engl J Med 1984;310:1477-80.

6. Hoff T. The new breed. Physician Executive 1996;23:31-6.

7. Colla CH, Lewis VA, Shortell SM, et al. First national survey of ACOs finds that physicians are playing strong leadership and ownership roles. Health Aff 2014;33:964-71.

8. Dwyer AJ. Medical managers in contemporary healthcare organisations: a consideration of the literature. Aust Health Rev 2010;34:514-22.

9. Bujak JS. Physicians in governance: their unique learning curve. Trustee 2005;58.30-28, 30.

10. Fulkerson W, Hartung D. Creating a healthy hospital: the demand for physician executives. Group Practice Journal 2006;55:41.

11. Ham C, Dickinson H. Engaging doctors in leadership: a review of the literature. NHS Institute for Innovation and Improvement, Coventry 2008.

12. Kippist L, Fitzgerald A. Organisational professional conflict and hybrid clinician managers: the effects of dual roles in Australian health care organizations. J Health Organ Manag 2009;23:642-55.

13. Mo TO, To M. Doctors as managers: moving towards general management? The case of unitary management reform in Norwegian hospitals. J Health Organ Manag 2008;22:400-15.

14. Loh E. Doctors as health managers: an oxymoron or a good idea?. Journal of Work-Applied Management 2015;7:52-60.

15. Dickinson H, Ham C, Snelling I, et al. Are we there yet? Models of medical leadership and their effectiveness: an exploratory study. NIHR Service Delivery and Organisation Programme 2013.

16. West M, Armit K, Loewenthal L, et al; Leadership and leadership development in healthcare: the evidence base. London: Faculty of medical leadership and management, 2015.

17. Schneller ES, Greenwald HP, Richardson ML, et al. The physician executive: role in the adaptation of American medicine. Health Care Manage Rev 1997;22:90-6.

18. Schultz FC, Pal S, Swan DA. Who should lead a healthcare organization: MDs or MBAs? J Healthc Manag 2004;49:103.

19. Clay-Williams R, Braithwaite J. Doctors in executive management: a systematic review of the peer-reviewed literature. Sydney, Australia: Centre for clinical governance research, Australian Institute of Health Innovation, University of New South Wales, 2012.

20. Braithwaite J, Westbrook MT. A survey of staff attitudes and comparative managerial and non-managerial views in a clinical directorate. Health Serv Manage Res 2004;17:141-66.

21. Davies HT, Hodges CL, Rundall TG. Views of doctors and managers on the doctor-manager relationship in the NHS. BMJ 2003;326:626-8. 
22. Elina V, Juhani L, Tiina TJ, et al. Doctor-managers as decision makers in hospitals and health centres. J Health Organ Manag 2006;20:85-94.

23. Spehar I, Frich JC, Kjekshus LE. Clinicians' experiences of becoming a clinical manager: a qualitative study. BMC Health Serv Res 2012;12:12:1.

24. Spehar I, Frich JC, Kjekshus LE. Clinicians in management: a qualitative study of managers' use of influence strategies in hospitals. BMC Health Serv Res 2014;14:14:1.

25. Martinussen PE, Magnussen J. Resisting market-inspired reform in healthcare: the role of professional subcultures in medicine. Soc Sci Med 2011;73:193-200.

26. Hawker S, Payne S, Kerr C, et al. Appraising the evidence: reviewing disparate data systematically. Qual Health Res 2002;12:1284-99.

27. Popay J, Roberts H, Sowden A, et al. Guidance on the conduct of narrative synthesis in systematic reviews. ESRC Methods Programme, UK National Centre for Research Methods 2006;1:b92.

28. Dickinson $\mathrm{H}$, Ham $\mathrm{C}$. Engaging doctors in leadership: review of the literature. Birmingham, University of Birmingham 2008.

29. Pihlainen V, Kivinen T, Lammintakanen J. Management and leadership competence in hospitals: a systematic literature review. Leadersh Health Serv 2016;29:95-110.

30. Sarto F, Veronesi G. Clinical leadership and hospital performance: assessing the evidence base. BMC Health Serv Res 2016;16(Suppl 2):169.

31. Agarwal R, Green R, Agarwal N, et al. Management practices in Australian healthcare: can NSW public hospitals do better? J Health Organ Manag 2016;30:331-53.

32. Goodall AH. Physician-leaders and hospital performance: is there an association? Soc Sci Med 2011;73:535-9.

33. Jiang $\mathrm{HJ}$, Lockee $\mathrm{C}$, Bass $\mathrm{K}$, et al. Board oversight of quality: any differences in process of care and mortality? J Healthc Manag 2009;54:15.

34. Konu A, Viitanen E. Shared leadership in Finnish social and health care. Leadersh Health Serv 2008;21:28-40.

35. Kuntz L, Scholtes S. Physicians in leadership: the association between medical director involvement and staff-to-patient ratios. Health Care Manag Sci 2013;16:129-38.

36. O'Keeffe ST. A cross-sectional study of doctors', managers' and public representatives' views regarding acceptable level of risk in discharges from the emergency department. QJM 2015;108:533-8.

37. Parayitam S, Phelps LD, Olson BJ. Strategic decision-making in the healthcare industry: the effects of physician executives on decision outcomes. Management Research News 2007;30:283-301.

38. Saleh S, Kaissi A, Semaan A, et al.Strategic planning processes and financial performance among hospitals in Lebanon. The International Journal of Health Planning and Management 2013.

39. Simonen O, Viitanen E, Lehto J, et al. Knowledge sources affecting decision-making among social and health care managers. $J$ Health Organ Manag 2009;23:183-99.

40. Bai G, Krishnan R. Do hospitals without physicians on the board deliver lower quality of care? Am J Med Qual 2015;30:58-65.

41. De Andrade Costa $L$. The effect of physician board membership on uncompensated care provision. Appl Econ 2014;46:2290-300.

42. Kuntz L, Pulm J, Wittland M. Hospital ownership, decisions on supervisory board characteristics, and financial performance. Health Care Manage Rev 2016;41:165-76.

43. Veronesi G, Kirkpatrick I, Vallascas F. Clinicians on the board: what difference does it make? Soc Sci Med 2013;77:147-55.
44. Spehar I, Frich JC, Kjekshus LE. Professional identity and role transitions in clinical managers. J Health Organ Manag 2015;29:353-66.

45. Prybil LD. Size, composition, and culture of high-performing hospital boards. Am J Med Qual 2006;21:224-9.

46. Lang TA, Hodge M, Olson V, et al. Nurse-patient ratios: a systematic review on the effects of nurse staffing on patient, nurse employee, and hospital outcomes. J Nurs Adm 2004;34:326-37.

47. Pronovost PJ, Angus DC, Dorman T, et al. Physician staffing patterns and clinical outcomes in critically ill patients: a systematic review. JAMA 2002;288:2151-62.

48. Braithwaite J, Clay-Williams R, Nugus P, et al; Health care as a complex adaptive system. In: Hollnagel E, Braithwaite J, Wears R, eds. Resilient health care. Surrey, UK: Ashgate Publishing Limited, 2013.

49. Goes JB, Zhan C. The effects of hospital-physician integration strategies on hospital financial performance. Health Serv Res 1995;30:507.

50. Molinari C, Alexander J, Morlock L, et al. Does the hospital board need a doctor? The influence of physician board participation on hospital financial performance. Med Care 1995;33:170-85.

51. Xirasagar S, Samuels ME, Curtin TF. Management training of physician executives, their leadership style, and care management performance: an empirical study. Am J Manag Care 2006;12:101-8.

52. Xirasagar S, Samuels ME, Stoskopf $\mathrm{CH}$. Physician leadership styles and effectiveness: an empirical study. Med Care Res Rev 2005;62:720-40.

53. Sebastian A, Fulop L, Dadich A, et al. Health LEADS Australia and implications for medical leadership. Leadersh Health Serv 2014;27:355-70.

54. Smits SJ, Bowden DE, Wells JO, et al. The role of the physician in transforming the culture of healthcare. Leadersh Health Serv 2016;29:300-12.

55. Mannion R, Davies HT, Marshall MN. Cultural characteristics of "high" and "low" performing hospitals. J Health Organ Manag 2005;19:431-9.

56. Quinn JF, Perelli S. First and foremost, physicians: the clinical versus leadership identities of physician leaders. J Health Organ Manag 2016;30:711-28.

57. Ramanuj PP, Ryland H, Mitchell EW, et al. In the spotlight: healthcare inspections as an opportunity for trainee clinicians to be the leaders of today. BMJ Qual Saf 2014;23:624-8.

58. Penlington C, Holmstrom K. Practitioner leadership: a missing link in leadership theory. The International Journal of Leadership in Public Services 2013:;9:32-46.

59. Ham C, Clark J, Spurgeon P, et al. Doctors who become chief executives in the NHS: from keen amateurs to skilled professionals. $J$ $R$ Soc Med 2011;104:113-9.

60. Kisa A, Ersoy $K$. The need for time management training is universal: evidence from Turkey. Hosp Top 2005;83:13-19.

61. West E, Barron DN. Social and geographical boundaries around senior nurse and physician leaders: an application of social network analysis. Can J Nurs Res 2005;37:132-49.

62. Creswick N, Westbrook J. Examining the socialising and problemsolving networks of clinicians on a hospital ward. Conference Proceedings of Social Science Methodology Conference of the Australian Consortium for Social and Political Research (ACSPR), 2006. 